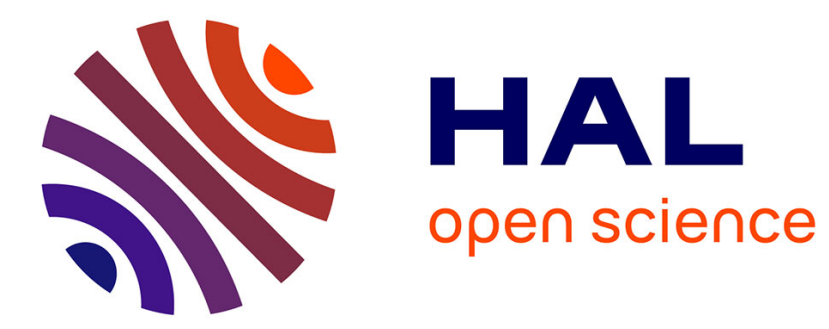

\title{
Sclérose en plaques : les nouvelles approches physiopathologiques
}

H. Zéphir

\section{To cite this version:}

H. Zéphir. Sclérose en plaques: les nouvelles approches physiopathologiques. Pratique Neurologique - FMC, 2019, 10, pp.112 - 117. 10.1016/j.praneu.2019.02.011 . hal-03486506

\section{HAL Id: hal-03486506 https://hal.science/hal-03486506}

Submitted on 20 Dec 2021

HAL is a multi-disciplinary open access archive for the deposit and dissemination of scientific research documents, whether they are published or not. The documents may come from teaching and research institutions in France or abroad, or from public or private research centers.
L'archive ouverte pluridisciplinaire HAL, est destinée au dépôt et à la diffusion de documents scientifiques de niveau recherche, publiés ou non, émanant des établissements d'enseignement et de recherche français ou étrangers, des laboratoires publics ou privés.

\section{다)(1) $(5$}

Distributed under a Creative Commons Attribution - NonCommercial| 4.0 International 


\title{
aSclérose en plaques : les nouvelles approches physiopathologiques Multiple sclerosis: new pathophysiological approaches
}

\author{
Pr Hélène Zéphir ${ }^{1,2} *$
}

${ }^{1}$ Service de Neurologie D-Clinique Neurologique-CHU de Lille-Université de Lille, 2 Avenue Oscar Lambret, 59000 Lille, France

${ }^{2}$ LIRIC, U 995, Université de Lille, France

*Auteur correspondant :

Pr Hélène Zéphir, Service de Neurologie D-Clinique Neurologique-CHU de Lille-Université de Lille, 2 Avenue Oscar Lambret, 59000 Lille, France

Email : helene.zephir@chru-lille.fr

Tel : + 33320446846

Fax +33320444484 
Résumé : La sclérose en plaques (SEP) est une maladie inflammatoire démyélinisante et neurodégénérative du système nerveux central d'étiologie inconnue, non curable aujourd'hui mais dont le contrôle thérapeutique repose sur des immunothérapies ciblant les acteurs inflammatoires du processus. Les progrès immunopathologiques, d'immunohistochimie mais aussi d'imagerie notamment les IRM de haute résolution et de haut champs définissent et précisent progressivement les mécanismes mis en jeu aux différents stades de la maladie. Ces outils tentent de combler l'espace entre la vision macroscopique de la clinique et de l'imagerie et la vision microscopique cellulaire, voire moléculaire. L'ensemble des confrontations clinico-IRM-immunopathologie rendent compte d'une maladie dont l'inflammation peut être focale mais également diffuse, plus ou moins compartimentée au cours de la maladie et faisant intervenir l'immunité adaptative et innée. La vision contemporaine de la SEP s'accompagne ainsi d'une considération clinique qui dépasse les différentes formes rémittente ou progressives. La SEP est aujourd'hui plutôt considérée (i) active ou en aggravation faisant référence à l'ensemble des processus inflammatoires mis en jeu par le système immunitaire adaptatif comme inné, (ii) progressive faisant référence au phénomène neurodégénératif secondaire à l'inflammation et ces dégâts, et d'ordre vraisemblablement oxydatif.

MOTS CLES : Sclérose en plaques, immunologie, IRM, microglie, inflammation 


\begin{abstract}
Multiple sclerosis (MS) is an inflammatory demyelinating and neurodegenerative disease of the central nervous system (CNS). Its etiology remains unknown and uncurable, but immunotherapies can control underlying inflammatory processes. Progress achieved in the fields of immunopathology, immunochemistry and high-resolution and high-field magnetic resonance imaging (MRI) define more precisely the different mechanisms involved in the different stages of the disease. Those tools can be used to try to fill in the gap between the macroscopic clinical and MRI findings and the cellular and even microscopic molecular information provided by immunochemistry. The results of clinical, MRI and immunological studies highlight a disease where the specific inflammatory processes can be focal, diffuse and potentially compartmentalized, notably over time, involving the innate as well as adaptative immune systems. The contemporary vision of MS is associated with a clinical view which goes beyond the phenotypic relapsing and progressive forms of the disease. As in Lublin's proposal, MS should be considered today as $i$ ) active or worsening referring to the inflammatory processes involving the adaptative and innate immune systems, $i$ ) progressive referring to neurodegenerative phenomena subsequent to inflammation leading to an oxidative burst with mitochondrial damage.
\end{abstract}

KEY WORDS : Multiple sclerosis, immunology, MRI, microglia, inflammation 
La sclérose en plaques (SEP) est une maladie chronique inflammatoire, démyélinisante et dégénérative dont les lésions mettent en avant des acteurs du système immunitaire macrophagiques essentiellement mais aussi lymphocytaires et plasmocytaires. Les lésions sont périveinulaires focales associées à de la démyélinisation et à une rupture de la barrière hémato-encépahlique (BHE). Il est aussi décrit sans rupture évidente de cette BHE, des infiltrats inflammatoires plus diffus et parfois compartimentés dans les formes progressives de la maladie avec des follicules lymphoïdes méningés. Une inflammation diffuse est par ailleurs décrite en substance blanche d'apparence normale où l'activation microgliale semble prédominer. Les immunothérapies validées efficaces aujourd'hui ciblent essentiellement les acteurs inflammatoires lymphocytaires et si les paramètres cliniques et IRM d'inflammation focale semblent être maitrisés en partie, le contrôle du processus neurodégénératif consécutif est incertain. Le déroulement des séquences inflammatoires et de ses conséquences restent à préciser et les progrès neuropathologiques, d'immunohistochimie et en IRM permettent de préciser progressivement les mécanismes et donc les cibles thérapeutiques potentielles dans le but d'un contrôle plus complet des processus pathologiques.

\section{Les lésions de SEP ne correspondent pas à une inflammation non spécifique}

La présence de larges lésions démyélinisantes et confluentes dans la substance grise et blanche du système nerveux central (SNC) sont la caractéristique de la maladie inflammatoire associant démyélinisation avec la destruction oligodendrocytaire [1]. Bien que la démyélinisation puisse être intense et totale, les axones sont préservés largement et la perte neuronale est variable d'un patient à l'autre et d'une lésion à l'autre chez un même patient [2,3]. Ces lésions surviennent dans un décor inflammatoire fait de macrophages, de lymphocytes $\mathrm{T}$, lymphocytes $\mathrm{B}$ et de plasmocytes $[1,4]$.

Cette inflammation débute près des veines post-capillaires. Les lésions démyélinisantes ainsi initialement périveineuses vont confluer et s'étendre vers la substance blanche d'apparence normale. La démyélinisation est associée à une activation astrocytaire au stade de lésion active pouvant aboutir à des lésions gliotiques cicatricielles et inactives. Les lésions de SEP peuvent en partie se remyéliniser par recrutement et différenciation de progéniteurs d'oligodendrocytes [5].

Ces même lésions sont aussi formées dans la substance grise corticales [6,7], mais aussi des noyaux gris centraux $[8,9]$ et de la moelle épinière [1]. Par ailleurs dans le cortex cérébral une démyélinisation corticale sous-piale est observée particulièrement dans les formes 
progressives de la maladie, associée à une infiltration inflammatoire des leptoméninges [7,10]. Les lésions s'étendent de la surface piale aux couches plus profondes du cortex. Elles peuvent être visibles à tous les stades et sont principalement localisées dans les sillons corticaux et les invaginations cérébrales comme le cortex insulaire et cingulaire. Les lésions corticales sous piales ne sont pas observées dans d'autres affections inflammatoires cérébrales touchant le cortex [11].

Cette inflammation n'est pas seulement focale et prononcée dans les lésions actives démyélinisantes mais aussi diffuses et visibles dans les plaques inactives et remyélinisées comme dans la substance blanche d'apparence normale [12].

Ainsi, la démyélinisation de la SEP est sélectivement périveinulaire et confluente avec une perte oligodendrocytaire et discrimine la SEP des autres maladies aux lésions focales de substance blanche et grise. Ainsi dans la neuromyélite optique de Devic, les lésions de démyélinisation associées à une destruction axonale sévère fait suite à des dommages astrocytaires primitifs liés à un anticorps spécifiques anti-aquaporine 4 [13]. Dans la leucoencephalopathie multifocale progressive la démyélinisation affecte initialement les oligodendrocytes uniquement sans la caractéristique périveinulaire de la SEP [14]. Les lésions d'autres maladies inflammatoires ou des accidents vasculaires cérébraux détruisent parallèlement la myéline, les oligodendrocytes et les axones. Dans les maladies chroniques telles que la tuberculeuse où les infiltrats méningées et corticaux ont une composition similaire à ce qui est observé dans le SEP, il n'y a pas d'infiltration sous piales. Il n'y en a pas non plus dans les encephalites paranéoplasiques. Par ailleurs il n'y pas de démyelinisation chez les patients ayant un lymphome B infiltrant les méninges [15].

\section{Approche tissulaire de la SEP en IRM}

\section{Topographie périveinulaire du développement des lésions de SEP}

La topographie périveinulaire du développement des lésions de SEP n'a pu être que récemment visualisée in vivo en utilisant des images IRM à haut champs (3T) ou ultra champs (7T) de susceptibilité magnétique en haute résolution [16,17]. La caractéristique du signe de la veine centrale dans la lésion de SEP est associée à une concentration élevée à ce niveau de deoxyhémoglobine (plus grande extraction d'oxygène sur le site de l'inflammation) et la variation du diamètre du vaisseau (flux veineux ralenti, processus de cicatrisation postinflammatoire de la paroi veineuse) [18,19]. 


\section{Lésions corticales}

Les lésions corticales bien que plus souvent observées dans les formes progressives, sont présentes à tous les stades de la maladie en pathologie et sont probablement sous estimées en IRM. Les séquences en double inversion-récupération, les séquences $\mathrm{T} 2 *$ en haute résolution et les séquences en transfert de magnétisation peuvent détecter certaines de ces lésions, particulièrement celles qui sont transcorticales ou les lésions appelées leuco-corticales intéressant à la fois le cortex et la substance blanche en regard. Les lésions sous-piales et les lésions intra-corticales de petite taille sont mieux visualisées sur les images post-mortem en haute résolution et à ultra champs, mais ne sont rapportées in vivo que sporadiquement $[20,21]$.

La barrière hémato-encéphalique (BHE) : plus ou moins perméable au cours de la chronicisation de l'inflammation

\section{La lésion inflammatoire active}

Les résultats de travaux à la fois humains et expérimentaux (EAE) sont cohérents dans la détection de subtiles modifications radiologiques dans la substance blanche d'apparence normale des jours ou des semaines avant le rehaussement par le gadolinium qui définit le début de la lésion de SEP [22-24]. Ces études supportent le concept d'une dissociation temporelle très courte entre les événements immunologiques initiateurs de la lésion et l'ouverture franche de la BHE et la démyélinisation secondaire. Récemment Absinta et al. visualisaient les altérations focales de signal en IRM (en T2-FLAIR et T2*) autour de la veine centrale de la lésion de SEP en devenir [25]. Avant le début manifeste de la démyélinisation radiologiquement définie, la veine centrale inflammée se rehausse isolément, possiblement par une modification de la perméabilité de la BE avec capture du produit de contraste dans les espaces périvasculaires [25].

\section{L'inflammation chronique}

Elle est compartimentée derrière une BHE non ouverte et donc reste invisible en IRM conventionnelle avec gadolinium. Les macrophages venant des monocytes périphériques marqués à l'USPIO (ultrasmall superparamagnetic iron oxide particles) peuvent être suivis en bordure des lésions démyélinisées alors que la BHE semble intact (c'est-à-dire des lésions non rehaussées par le gadolinium) [26-28]. L'utilisation de l'imagerie de susceptibilité en haute résolution et à haut champs a récemment démontré la possibilité de détecter à la marge de 
lésions non rehaussées par le gadolinium, un anneau paramagnétique. La production de radicaux libres et/ou l'accumulation de fer en intracellulaire associés à l'accumulation régulière de macrophages en bord de lésions peut expliquer le signal paramagnétique observé.

\section{Place du système immunitaire inné}

L'implication des lymphocytes et du système immunitaire adaptatif est largement reconnu, alors que les cellules myéloïdes issues du système immunitaire inné et qui incluent les monocytes, les macrophages, les cellules dendritiques et la microglie sont communément moins reliés à la maladie. Pourtant, les macrophages et la microglie sont les principales cellules inflammatoires dans les plaques de SEP actives ou chroniques, et elles persistent dans les formes secondairement progressives de SEP. Ces cellules myéloïdes ont des propriétés qui dépassent largement leur capacité de présentation d'antigène. Elles peuvent produire des cytokines pro-inflammatoires, des radicaux libres, des protéases et d'autres médiateurs qui peuvent entrainer des dégâts tissulaires dans la SEP. Par ailleurs leur interaction avec le système lymphoïde majore et pérennise 1 'environnement inflammatoire aboutissant à la destruction oligodendroctaire, la démyélinistaion et la mort neuronale [29].

\section{Monocytes}

Les monocytes circulants sont classés en 2 groupes. Les monocytes immatures proinflammatoires sont retrouvés dans les lésions où l'environnement tissulaire permet leur différenciation tissulaire en macrophages pro-inflammatoires [30,31]. D'autres monocytes circulants sont en patrouille et potentiellement régulateurs et peuvent devenir des macrophages résidents tissulaires qui peuvent aller en condition saine dans les espaces périvasculaires entre l'endothélium et les membranes basales des veines post-capillaires [31]. Les leucocytes se mobilisent dans ces espaces périvasculaires avant de passer la BHE [32,33]. Dans l'encéphalite autoimmune expérimentale (EAE), la déplétion des monocytes et des macrophages bloque l'invasion du SNC par les lymphocytes. Ainsi les macrophages périvasculaires pourraient détecter les antigènes dans cet espace périvasculaires et activer les lymphocytes qui pénètrent ensuite le système nerveux central [34]. D'autres populations de macrophages observées dans du cerveau non malade inclus les macrophages méningés et les macrophages des plexus choroïdes, ces derniers détecteraient les désordres de la BHE liés aux lésions ou aux infections. 


\section{Macrophages et microglie}

Comme les monocytes, les macrophages et la microglie peuvent être classés en fonction de leur polarité [35]. Deux sous populations majeures de macrophages ont été définies: les macrophages « M1 » (activation classique) et les macrophages «M2 » (activation alternative). L'interféron (IFN)- $\gamma$ et le lipopolysaccharide sont les activateurs des macrophages M1. La production de l'enzyme synthétase d'oxyde d'azote est alors induite aboutissant à des niveaux élevés d'oxyde d'azote. Les macrophages M1 produisent de grande quantité d'IL-1b, IL 12 et du Tumor Necrosis Factor (TNF) qui sont largement pro-inflammatoires.

Les macrophages M2 sont activés par les dommages tissulaires locaux ou l'infestation parasitaire. In vitro les macrophages M2 peuvent être activés par l'interleukine 4 et 13, l'arginine utilisée dans les substrats de culture tissulaire aide alors à former les polyamines, la proline et le collagène nécessaires à la réparation des lésions [35]. Les macrophages M2 sécrètent des cytokines régulatrices ou anti-inflammatoires telles que l'antoagoniste au récepteur de l'interleukine 4, l'antagoniste de récepteur à l'Interleukine 1.

Cette classification en M1 et M2 des macrophages soulignent les différences fonctionnelles, mais cette subdivision est vraisemblablement trop simplifiée. En effet, l'analyse transcriptomique (donc de l'expression des gènes mis en jeu) des macrophages humains activés par différents stimuli révèlent au moins 9 différentes variétés de cellules [36].

\section{Cellules dendritiques}

Les cellules dendritiques conventionnelles (sont ?)des cellules myéloides primordiales pour activer les lymphocytes $\mathrm{T}$ à la fois dans des conditions saines et dans des conditions d'activation pathogénique.

\section{Que nous apprenne les études pathologiques?}

Les études autopsiques ont révélé dans la substance blanche d'apparence normale de sujets ayant une SEP, que l'extension de l'inflammation microgliale locale est corrélée à l'extension de la perturbation paranodale [37]. Kutzelnigg en 2005 montrait que la démyélinisation corticale et la perte axonale diffuse dans la substance blanche d'apparence normale était associés à une activation microgliale profonde [6]. Prineas décrivait dès 2001 dans les formes progressives de SEP des agrégats de cellules microgliales majoritaires dans les lésions [38]. Vogel en 2013 démontre qu'au cœur d'une lésion active de SEP, les macrophages et la glie emplis de débris myéliniques, expriment à leur surface aussi bien les marqueurs cellulaires d'inflammation que de régulation [39]. Ces nombreuses cellules au sein des lésions de SEP 
peuvent donc avoir un statut d'activation soit pro-inflammatoire soit pro-régulateur. Peferoen ajoute enfin que dans les lésions préactives et remyélinisantes de SEP, des macrophages exprimaient à la fois les marqueurs M1 et ceux de M2 (2015). Dans cette étude, in vitro en tout cas, une microglie polarisée dans une direction pouvait changer totalement de polarité si elle était exposée à un environnement cytokinique opposée à la première polarisation. Enfin l'interaction entre lymphocytes $\mathrm{T}$ et cellules myéloïdes semblent passer par le GM-CSF (granulocyte macrophage colony-stimulating factor) qui est un pré-requis pour le développement de l'EAE [40]. Le GM-CSF produit par les lymphocytes T encéphalitogènes dans l'EAE induisent l'expression de chémokines par des monocytes porteurs des récepteurs à ces chemokines inflammatoires pour l'activation de l'inflammasome dans les mêmes cellules T aboutissant à une pérennisation de l'inflammation et des dégâts tissulaires.

\section{Que nous apportent les études en imagerie?}

L'imagerie en PET 11C-PK11195 de la microglie, montre que l'activité microgliale dans la substance grise des patients ayant une SEP est corrélée à la progression du handicap [41]. Par ailleurs l'activation microgliale peut être détectée dans la substance blanche d'apparence normale avant l'infiltration leucocytaire, la démyélinisation ou la rupture de la barrière hémato-encéphalique [42]. Les patients qui présentent une activation microgliale majeure sont ceux qui présentent les lésions corticales [43].

\section{Les traitements agissent-ils sur la lignée myéloïde?}

L'ensemble de ces données suggèrent ainsi que l'inhibition de la microglie neurotoxique pourrait être une piste thérapeutique potentielle dans la SEP. Aujourd'hui l'arsenal thérapeutique développé ne comprend que des molécules dont les capacités à traverser la BHE est restreinte. Les immunothérapies utilisées ciblent les lymphocytes T et/ou B mais elles peuvent néanmoins aussi affecter les cellules myéloïdes.

Les monocytes de patients traités par IFN $\beta$ produisent moins de cytokines proinflammatoires (IL12) et plus de cytokines anti-inflammatoires (IL10) que les monocytes de patients non traités, suggérant que les cellules myéloïdes passent sous traitement vers un profil régulateur $[44,45]$. L'acétate de glatiramer permet la genèse de cellules myéloïdes régulatrices (appelées aussi cellules présentatrices d'antigène de type II) qui seraient responsables de la genèse des lymphocytes Th2 anti-inflammatoires [46]. Le récepteur au sphingosine-1 phosphate (S1P) est présent sur de nombreuses cellules immunitaires et les cellules myéloïdes. Le traitement par fingolimod ne semble pas réduire le nombre absolu de 
monocytes et de cellules dendritiques mais diminue la capacité de ces cellules à produire des cytokines pro-inflammatoires [47]. Si le diméthylfumarate est associé à une diminution du taux de lymphocytes dans les 12 premiers mois de traitement, il n'y a pas de variation du nombre de monocytes. Une étude publiée en 2016 montrait que les monocytes de patients traités par diméthylfumarate exprimaient plus faiblement miR-155 (micro-ARN inflammatoire) que les monocytes de patients non traités [48]. Le natalizumab ne se lie pas seulement aux $\alpha 4$-intégrines des lymphocytes mais aussi sur ceux des cellules myéloïdes bloquant ainsi leur migration vers le système nerveux central. Les patients traités par natalizumab ont après 24 mois de traitement des taux de lymphocytes circulants et des taux de monocytes circulants plus élevés. Le phénotype de ces nombreux monocytes n'a pas été exploré. Le CD 52 ciblé par l'alemtuzumab se trouve sur de nombreux leucocytes mais pas sur le monocyte dont il n'existe donc pas de déplétion sous ce traitement [49]. Les modifications de l'activité cellulaire myéloïde sous alemtuzumab n'est pas connu. Enfin l'effet de la déplétion cellulaire B est aujourd'hui le meilleur argument prouvant le rôle clef des lymphocytes B dans la physiopathogénie de la SEP. L'effet des anti-CD 20 sur la population myéloïde n'est pas connue.

\section{Démyélinisation et neurodégénescence finale}

L'étude des gènes dans les lésions actives corticales de SEP a montré une expression différentielle de gènes par rapport aux populations contrôles neurodégénératives (maladie d'Alzheimer) et contrôles sains [15]. Dans les plaques actives les protéines codées étaient associées à une explosion oxydative de la microglie, des dégâts mitochondriaux et de la neurodégénérescence [15]. Ces anomalies différentielles étaient validées en analyse pathologique et imunohistochimie. L'oxydation lipidique intense retrouvée correspond à des désordres mitochondriaux et l'observation de cellules en déficience respiratoire mais aussi l'accumulation de délétions génétiques mitochondriales [50,51]. Le désordre mitochondrial semble être au centre de la cascade pathogénique conduisant à «l'hypoxie virtuelle » [52]. La conséquence en est le déficit énergétique résultant du déséquilibre ionique cellulaire et la neurodégénérescence cellulaire calcium-dépendant [53]. Haider et al souligne par ailleurs l'amplification du phénomène oxydatif pathologique dans les phases progressives de la maladie en raison du cumul lésionnel mais aussi par vieillissement cérébral [54].

\section{Conclusion}


Parmi les nouvelles pistes physiopathologiques dans la SEP, le système immunitaire inné semble être un acteur majeur dans l'initiation des lésions et leur développement. Les mécanismes inflammatoires conduisent à une explosion oxydative caractéristique et en lien avec une hypoxie et un déficit énergétique par désordre mitochondrial, phénomène amplifié par le vieillissement cérébral.

\section{Liens d'intérêts}

Les auteurs n'ont pas transmis leurs liens d'intérêts éventuels. 


\section{Points essentiels}

- La démyélinisation et la neurodégénerescence sont une conséquence non spécifique d'un processus inflammatoire qui lui est en revanche spécifique de la SEP.

- Le faible nombre de lymphocytes aux sites de démyélinisations soutient un rôle direct ou indirect de l'activation microgliale.

- La démyélinisation et la neurodégénérescence semblent le résultat d'une cascade de réactions oxydatives, de dommages mitochondriaux, d'hypoxie et les conséquences moléculaires. Ces phénomènes sont amplifiés par à la fois l'accumulation lésionnelle et le vieillissement neuronal et cérébral.

- Les progrès en imagerie notamment avec les séquences d'IRM en haute résolution et les ultra-champs (7T) permettent d'améliorer l'abord diagnostique mais aussi de préciser et de suivre les processus inflammatoires plus ou moins compartimentées. 


\section{Références}

[1] Lassmann H. Multiple Sclerosis Pathology.Cold Spring Harb Perspect Med. 2018 Mar $1 ; 8(3)$.

[2] Trapp BD, Peterson J, Ransohoff RM, Rudick R, Mörk S, Bö L. Axonal transection in the lesions of multiple sclerosis. N Engl J Med. 1998;338:278-85.

[3]Ferguson B, Matyszak MK, Esiri MM, Perry VH. Axonal damage in acute multiple sclerosis lesions. Brain. 1997;120:393-9.

[4] Charcot JM.1880. Leçons sur les maladies du systèmenerveux faites à la Salpétrière [lectures about the diseases of the nervous central system done at the Salpétrière], 4th ed., Vol. IA. Delahaye, Paris.

[5] Prineas JW, Barnard RO, Kwon EE, Sharer LR, Cho ES. Multiple sclerosis: remyelination of nascent lesions. Ann Neurol. 1993;33:137-51.

[6] Kutzelnigg A, Lucchinetti CF, Stadelmann C, Brück W, Rauschka H, Bergmann M, Schmidbauer M, Parisi JE, Lassmann H. Cortical demyelination and diffuse white matter injury in multiple sclerosis. Brain. 2005;128:2705-12.

[7] Kutzelnigg A, Faber-Rod JC, Bauer J, Lucchinetti CF, Sorensen PS, Laursen H, Stadelmann C, Brück W, Rauschka H, Schmidbauer M, Lassmann H. Widespread demyelination in the cerebellar cortex in multiple sclerosis. Brain Pathol. 2007;17:38-44.

[8] Cifelli A, Arridge M, Jezzard P, Esiri MM, Palace J, Matthews PM. Thalamic neurodegeneration in multiple sclerosis.Ann Neurol. 2002;52:650-3.

[9]Haider L, Simeonidou C, Steinberger G, Hametner S, Grigoriadis N, Deretzi G, Kovacs GG, Kutzelnigg A, Lassmann H, Frischer JM. Multiple sclerosis deep grey matter: the relation between demyelination, neurodegeneration, inflammation and iron. J Neurol Neurosurg Psychiatry. 2014;85:1386-95.

[10] Bø L, Vedeler CA, Nyland HI, Trapp BD, Mørk SJ. Subpial demyelination in the cerebral cortex of multiple sclerosis patients. J Neuropathol Exp Neurol. 2003;62:723-32.

[11] Moll NM ${ }^{1}$, Rietsch AM, Ransohoff AJ, Cossoy MB, Huang D, Eichler FS, Trapp BD, Ransohoff RM. Cortical demyelination in PML and MS: Similarities and differences. Neurology. 2008;70:336-43.

[12] Frischer JM ${ }^{1}$, Bramow S, Dal-Bianco A, Lucchinetti CF, Rauschka H, Schmidbauer M, Laursen H, Sorensen PS, Lassmann H. The relation between inflammation and neurodegeneration in multiple sclerosis brains. Brain. 2009;132:1175-89.

[13] Misu T, Höftberger R, Fujihara K, Wimmer I, Takai Y, Nishiyama S, Nakashima I, Konno H, Bradl M, Garzuly F, Itoyama Y, Aoki M, Lassmann H. Presence of six different lesion types suggests diverse mechanisms of tissue injury in neuromyelitis optica. Acta Neuropathol. 2013;125:815-27.

[14]Bauer J, Gold R, Adams O, Lassmann H. Progressive multifocal leukoencephalopathy and immune reconstitution inflammatory syndrome (IRIS). Acta Neuropathol. 2015;130:75164.

[15] Fischer MT, Wimmer I, Höftberger R, Gerlach S, Haider L, Zrzavy T, Hametner S, Mahad D, Binder CJ, Krumbholz M, Bauer J, Bradl M, Lassmann H. Disease-specific molecular events in cortical multiple sclerosis lesions. Brain. 2013;136:1799-815. 
[16] Hammond KE, Metcalf M, Carvajal L, Okuda DT, Srinivasan R, Vigneron D, Nelson SJ, Pelletier D. Quantitative in vivo magnetic resonance imaging of multiple sclerosis at 7 Tesla with sensitivity to iron. Ann Neurol. 2008 ;64:707-13.

[17] Absinta M, Sati P, Gaitán MI, Maggi P, Cortese IC, Filippi M, Reich DS. Seven-tesla phase imaging of acute multiple sclerosis lesions: a new window into the inflammatory process. Ann Neurol. 2013;74:669-78.

[18] Gaitán MI, Maggi P, Wohler J, Leibovitch E, Sati P, Calandri IL, Merkle H, Massacesi L, Silva AC, Jacobson S, Reich DS. Perivenular brain lesions in a primate multiple sclerosis model at 7-tesla magnetic resonance imaging. Mult Scler. 2014;20:64-71.

[19] Dal-Bianco A ${ }^{1}$, Hametner S, Grabner G, Schernthaner M, Kronnerwetter C, Reitner A, Vass C, Kircher K, Auff E, Leutmezer F, Vass K, Trattnig S. Veins in plaques of multiple sclerosis patients - a longitudinal magnetic resonance imaging study at 7 Tesla. Eur Radiol. 2015;25:2913-20

[20] Mainero C, Benner T, Radding A, van der Kouwe A, Jensen R, Rosen BR, Kinkel RP. In vivo imaging of cortical pathology in multiple sclerosis using ultra-high field MRI.

Neurology. 2009;73:941-8.

[21] Nielsen AS, Kinkel RP, Madigan N, Tinelli E, Benner T, Mainero C. Contribution of cortical lesion subtypes at 7T MRI to physical and cognitive performance in MS.

Neurology. 2013;81:641-9.

[22] Filippi M, Mastronardo G, Rocca MA, Capra R, Gasperini C, Rovaris M, Bastianello S, Comi G. Detecting new lesion formation in multiple sclerosis: the relative contributions of monthly dual-echo and T1-weighted scans after triple-dose gadolinium. Eur Neurol. 1998;40:146-50.

[23] Werring DJ, Brassat D, Droogan AG, Clark CA, Symms MR, Barker GJ, MacManus DG, Thompson AJ, Miller DH. The pathogenesis of lesions and normal-appearing white matter changes in multiple sclerosis: a serial diffusion MRI study. Brain. 2000;123:1667-76.

[24] Wuerfel J, Bellmann-Strobl J, Brunecker P, Aktas O, McFarland H, Villringer A, Zipp F. Changes in cerebral perfusion precede plaque formation in multiple sclerosis: a longitudinal perfusion MRI study. Brain. 2004;127:111-9.

[25] Absinta M, Nair G, Sati P, Cortese IC, Filippi M, Reich DS. Direct MRI detection of impending plaque development in multiple sclerosis. Neurol Neuroimmunol

Neuroinflamm. 2015;2:e145.

[26] Vellinga MM, Oude Engberink RD, Seewann A, Pouwels PJ, Wattjes MP, van der Pol SM, Pering C, Polman CH, de Vries HE, Geurts JJ, Barkhof F. Pluriformity of inflammation in multiple sclerosis shown by ultra-small iron oxide particle enhancement.Brain. 2008;131:800-7.

[27] Tourdias T, Roggerone S, Filippi M, Kanagaki M, Rovaris M, Miller DH, Petry KG, Brochet B, Pruvo JP, Radüe EW, Dousset V. Assessment of disease activity in multiple sclerosis phenotypes with combined gadolinium- and superparamagnetic iron oxide-enhanced MR imaging. Radiology. 2012;264:225-33.

[28] Maarouf A, Ferré JC, Zaaraoui W, Le Troter A, Bannier E, Berry I, Guye M, Pierot L, Barillot C, Pelletier J, Tourbah A, Edan G, Audoin B, Ranjeva JP. Ultra-small superparamagnetic iron oxide enhancement is associated with higher loss of brain tissue structure in clinically isolated syndrome. Mult Scler. 2016;22:1032-9. 
[29] Mishra MK, Yong VW. Myeloid cells - targets of medication in multiple sclerosis. Nat Rev Neurol. 2016;12:539-51.

[30] Geissmann F, Gordon S, Hume DA, Mowat AM, Randolph GJ. Unravelling mononuclear phagocyte heterogeneity. Nat Rev Immunol. 2010;10:453-60.

[31] Gordon S, Taylor PR. Monocyte and macrophage heterogeneity. Nat Rev Immunol. 2005;5:953-64.

[32] Sorokin L. The impact of the extracellular matrix on inflammation. Nat Rev Immunol. 2010;10:712-23.

[33] Agrawal SM, Williamson J, Sharma R, Kebir H, Patel K, Prat A, Yong VW. Extracellular matrix metalloproteinase inducer shows active perivascular cuffs in multiple sclerosis. Brain. 2013;136:1760-77.

[34] Tran EH, Hoekstra K, van Rooijen N, Dijkstra CD, Owens T. Immune invasion of the central nervous system parenchyma and experimental allergic encephalomyelitis, but not leukocyte extravasation from blood, are prevented in macrophage-depleted mice. J Immunol. 1998;161:3767-75.

[35] Gordon $\mathrm{S}^{1}$, Martinez FO. Alternative activation of macrophages: mechanism and functions.. Immunity. 2010;32:593-604.

[36] Xue J, Schmidt SV, Sander J, Draffehn A, Krebs W, Quester I, De Nardo D, Gohel TD, Emde M, Schmidleithner L, Ganesan H, Nino-Castro A, Mallmann MR, Labzin L, Theis H, Kraut M, Beyer M, Latz E, Freeman TC, Ulas T, Schultze JL. Transcriptome-based network analysis reveals a spectrum model of human macrophageactivation. Immunity. 2014;40:27488 .

[37] Howell OW, Rundle JL, Garg A, Komada M, Brophy PJ, Reynolds R. Activated microglia mediate axoglial disruption that contributes to axonal injury in multiple sclerosis. $\mathbf{J}$ Neuropathol Exp Neurol. 2010

[38] Prineas JW ${ }^{1}$, Kwon EE, Cho ES, Sharer LR, Barnett MH, Oleszak EL, Hoffman B, Morgan BP. Immunopathology of secondary-progressive multiple sclerosis. Ann Neurol. 2001;50:646-57.

[39] Vogel DY, Vereyken EJ, Glim JE, Heijnen PD, Moeton M, van der Valk P, Amor S, Teunissen CE, van Horssen J, Dijkstra CD. Macrophages in inflammatory multiple sclerosis lesions have an intermediate activation status. J Neuroinflammation. 2013;10:35.

[40] El-Behi M $^{1}$, Ciric B, Dai H, Yan Y, Cullimore M, Safavi F, Zhang GX, Dittel BN, Rostami A.The encephalitogenicity of T(H)17 cells is dependent on IL-1- and IL-23induced production of the cytokine GM-CSF. Nat Immunol. 2011;12:568-75

[41] Politis M, Piccini P. In vivo imaging of the integration and function of nigral grafts in clinical trials. Prog Brain Res. 2012;200:199-220.

[42] van Noort JM, van den Elsen PJ, van Horssen J, Geurts JJ, van der Valk P, Amor S. Preactive multiple sclerosis lesions offer novel clues for neuroprotective therapeutic strategies. CNS Neurol Disord Drug Targets. 2011;10:68-81.

[43] Kooi EJ, Strijbis EM, van der Valk P, Geurts JJ. Heterogeneity of cortical lesions in multiple sclerosis: clinical and pathologic implications. Neurology. 2012;79:1369-76.

[44] Liu Z, Pelfrey CM, Cotleur A, Lee JC, Rudick RA. Immunomodulatory effects of interferon beta-1a in multiple sclerosis. J Neuroimmunol. 2001;112:153-62. 
[45] Hussien $Y^{1}$, Sanna A, Söderström M, Link H, Huang YM. Multiple sclerosis: expression of CD1a and production of IL-12p70 and IFN-gamma by blood mononuclear cells in patients on combination therapy with IFN-beta and glatiramer acetate compared to monotherapy with IFN-beta. Mult Scler. 2004;10:16-25.

[46] Weber MS, Hohlfeld R, Zamvil SS. Mechanism of action of glatiramer acetate in treatment of multiple sclerosis. Neurotherapeutics. 2007;4:647-53.

[47] Luessi F, Kraus S, Trinschek B, Lerch S, Ploen R, Paterka M, Roberg T, Poisa-Beiro L, Klotz L, Wiendl H, Bopp T, Jonuleit H, Jolivel V, Zipp F, Witsch E. FTY720 (fingolimod) treatment tips the balance towards less immunogenic antigen-presenting cells in patients with multiple sclerosis. Mult Scler. 2015;21:1811-22.

[48] Michell-Robinson MA, Moore CS, Healy LM, Osso LA, Zorko N, Grouza V, Touil H, Poliquin-Lasnier L, Trudelle AM, Giacomini PS, Bar-Or A, Antel JP. Effects of fumarates on circulating and CNS myeloid cells in multiple sclerosis. Ann Clin Transl Neurol. 2015 Dec 2;3(1):27-41.

[49] Coles $\mathrm{AJ}^{1}$, Cox A, Le Page E, Jones J, Trip SA, Deans J, Seaman S, Miller DH, Hale G, Waldmann H, Compston DA. The window of therapeutic opportunity in multiple sclerosis: evidence from monoclonal antibody therapy. J Neurol. 2006 Jan;253(1):98-108. Epub 2005 Jul 27.

[50] Mahad D, Ziabreva I, Lassmann H, Turnbull D. Mitochondrial defects in acute multiple sclerosis lesions. Brain. 2008;131:1722-35.

[51] Campbell GR, Ziabreva I, Reeve AK, Krishnan KJ, Reynolds R, Howell O, Lassmann H, Turnbull DM, Mahad DJ. Mitochondrial DNA deletions and neurodegeneration in multiple sclerosis. Ann Neurol. 2011;69:481-92.

[52] Mahad DH, Trapp BD, Lassmann H. Pathological mechanisms in progressive multiple sclerosis. Lancet Neurol. 2015;14:183-93.

[53] Stys PK, Zamponi GW, van Minnen J, Geurts JJ. Will the real multiple sclerosis please stand up? Nat Rev Neurosci. 2012;13:507-14.

[54] Haider L, Zrzavy T, Hametner S, Höftberger R, Bagnato F, Grabner G, Trattnig S, Pfeifenbring S, Brück W, Lassmann $\mathrm{H}$. The topograpy of demyelination and neurodegeneration in the multiple sclerosis brain. Brain. 2016;139:807-15. 